\title{
Application of Complex Network Principles to Key Station Identification in Railway Network Efficiency Analysis
}

\author{
Li Wang $\mathbb{D}^{1},{ }^{1}$ Min An $\mathbb{D}^{2},{ }^{2}$ Limin Jia $\mathbb{D}^{3},{ }^{3}$ and Yong Qin $\mathbb{D}^{3}$ \\ ${ }^{1}$ School of Traffic and Transportation, Beijing Jiaotong Univeristy, Beijing 100044, China \\ ${ }^{2}$ School of Science, Engineering and Environment, University of Salford, Manchester M5 4WT, UK \\ ${ }^{3}$ Rail Traffic Control and Safety State Key Laboratory, Beijing Jiaotong University, Beijing 100044, China
}

Correspondence should be addressed to Min An; m.an@salford.ac.uk

Received 31 January 2019; Revised 27 July 2019; Accepted 27 August 2019; Published 2 December 2019

Academic Editor: Paola Pellegrini

Copyright (c) 2019 Li Wang et al. This is an open access article distributed under the Creative Commons Attribution License, which permits unrestricted use, distribution, and reproduction in any medium, provided the original work is properly cited.

\begin{abstract}
Network efficiency analysis becomes important in railways in order to contribute towards improving the safety and capacity of the rail network, making rail travel more attractive for passengers, and improving industry practice and informing policy development. However, a physical railway network structure is a complicated system, and the operation, maintenance, and management of such a network is a difficult task which may be affected by many influential factors. By using efficiency analysis technology for a railway network, combining physical structure with operation functions can help railway industry to optimize the railway network while improving its efficiency and reliability. This paper presents a new methodology based on complex network principles that combines the physical railway structure with railway operation strategy for a railway network efficiency analysis. In this method, two network models of railway physical and train flow networks are developed for the identification of key stations in the railway network based on network efficiency contribution in which the terms of degree, strength, betweenness, clustering coefficient, and a comprehensive factor are taken into consideration. Once the key stations have been identified and analysed, the railway network efficiency is then studied on the basis of selective and random modes of the station failures. A case study is presented in this paper to demonstrate the application of the proposed methodology. The results show that the identified key stations in the railway network play an important role in improving the overall railway network efficiency, which can provide useful information to railway designers, engineers, operators and maintainers to operate and maintain railway network effectively and efficiently.
\end{abstract}

\section{Introduction}

In comparison with road transportation, railways are by far one of the safest means of ground transportation, especially for their passengers and employees. However, there are some issues involved in both maintaining this position in reality and sustaining the public perception of railway safety excellence. The railway now finds itself in a situation where actual and perceived safeties are real issues, to be dealt with in a new public culture of rapid change, short-term pressures, and instant communications [1-3]. However, operation and maintenance of railway networks are becoming difficult, particularly, it is related to railway network efficiency, reliability, and safety. For example, if a key station fails to operate in a railway network, it would affect the overall railway network transportation efficiency. A station failure to operate can be classified into physical failures of the railway physical network such as failures of signal system, power supply system, track system, and vehicle system, and the function failures of the train flow network such as inaccurate train route planning and scheduling $[4,5]$. A network efficiency is related to its demands, flows, costs, and behaviour on the network, which can be used to measure the importance of network components and their rankings $[6,7]$. In this study, network efficiency measure is used to assess the importance of railway network stations and their rankings, which is discussed in Section 4 and expressed in Equation (8). Based on the definition of network efficiency, therefore, the identification of key stations in the railway network efficiency analysis is the major task in the railway development and maintenance activities $[8,4]$. The railway network is a complex system with hundreds of stations and correlative operations. Therefore, the network efficiency analysis is becoming more important in aspect of ensuring safe and reliable operation to be applied in the railway network. This paper 
presents a new methodology to analyse the efficiency of the railway networks using complex network principles for the key station identification, in which railway network efficiency is evaluated based on station failure modes. This will provide useful method and information to the industry in the design, operation, and maintenance of the railway network effectively and efficiently.

In the context of the network theory, a complex network can be defined as a graph that is composed of relatively many mutually related nodes including structural and functional relations, and it could also be defined as a network that has nonobservable topological features that do not arise in simple networks such as random ones but often occur in graph models of real systems [9]. A railway network can be classified as a complex network and investigated through complex network analysis $[4,5]$. Complex network anlaysis has been successfully applied to analyse the efficiency of networks, for example, a biology network [10], a research cooperating network [11, 12], an electricity supply system [13], a traffic network [4, 14], and even an Internet network [15]. Dey et al. [16] successfully applied complex network theory to analyse safety and reliability of topology impact on the propagation of cascading failure in a national power grid. Zio and Sansavini [17] also applied complex network method for modelling interdependent network systems in order to identify cascade-safe operating margins. These researchers have developed various network analysis models and also studied the structural characteristics of the networks including system indicators such as node degree, length of the path, and clustering coefficient, in which the complex network vulnerability can be analysed in the selective and random node failure modes [18-21]. In the literature, some of the studies have also been conducted to investigate characteristics of transportation networks based on complex network theory. Xu et al. [22] studied urban bus transport systems, Porta et al. [23] and Wang et al. [7] looked at insight of urban street networks, Bagler [24] studied airline network, and Dall'Asta et al. [25] investigated USA airline network. Guidotti et al. [26] proposed a probabilistic methodology to quantify the network reliability based on current network efficiency and a measure of connectivity, i.e., eccentricity and heterogeneity. This method was applied to analyse a highway transportation network reliability. Qian et al. [27] developed a cascading failure model of the complex network to simulate the road traffic status by using the delay of the time, incident dissipation factor and load capacity. Chen et al. [28] presented a particle swarm optimization (PSO) algorithm to optimize the invulnerability of China railway traffic network by introducing the concept of the edge to the network. The results produced from these researches provide useful information for maintaining and operating of the complex road and airspace transportation networks.

Because a railway network is a complex network that is also suitable to be examined by using complex network principles. Lin et al. [29] and Li et al. [8] studied China's high-speed rail network as a complex system and analysed network safety and reliability based on complex network theory. Ouyang et al. [5] also applied complex network theory to study the performance and vulnerability of railways under various types of attacks and hazards. The complex network theory has been widely applied to the reliability and safety analysis of the complex networks and has also been used in railway network reliability analysis. However, these studies are limited to properties of the physical networks, and the railway operation functions are neglected in their analyses. It is essential to develop new methods and models to take not only the characteristics of physical railway network, but also the operation functions into considerationl in this case, train flow network needs to be integrated into the network efficiency analysis process in order to obtain reliable results.

This paper presents a new methodology to analyse railway network efficiency that combines characteristics of railway physical network with the functions of train flow network. In other words, the proposed method considers not only the physical network topologies such as degree and clustering coefficient, but also the dynamic operation parameters such as train running paths, stop-schedules, and service frequencies. The proposed method can be used to identify the key stations in the network and analyse railway network efficiency based on selective and random failures of stations, which provides a useful method and more reliable and accurate information to railway designers, engineers, operators, and maintainers for operating and maintaining railway network effectively and efficiently.

This paper is organised into the following sections. After the introduction, Section 2 presents the development of railway physical network (RPN) and train flow network (TFN) and considerations in the RPN and TFN are discussed more detailed. Specific terms in mathematics are defined in Section 3, which will form the basis of the proposed method using complex network principles for key station identification in the railway network. Section 4 describes a new proposed network efficiency analysis method. A case study of a highspeed rail network efficiency analysis is presented in Section 5 to demonstrate the application of the proposed method, and recommendations are given in this section for improvement of railway network at planning, operation and maintenance in order to satisfy the railway network efficiency requirement. Finally, conclusions are given in Section 6.

\section{Development of Railway Physical Network (RPN) and Train Flow Network (TFN)}

A new approach for the analysis of network efficiency is proposed, which combines the RPN with the TFN in order to consider network structure properties together with network operation functions in the analysis process. The RPN considers the physical connecting properties in the network and provides constraints to the TFN. The RFN takes train service plan and the operation functions of TFN into consideration. The proposed railway network efficiency analysis process includes three steps, i.e., development of railway network efficiency model, identification of key station indices, station ranking, and network efficiency analysis as shown in Figure 1.

A RPN can be developed based on the existing railway network, and then a TFN of a service plan can be established by integrating RPN into operation strategies such as train running routes, stop-schedules and service frequencies $[4,26]$. 


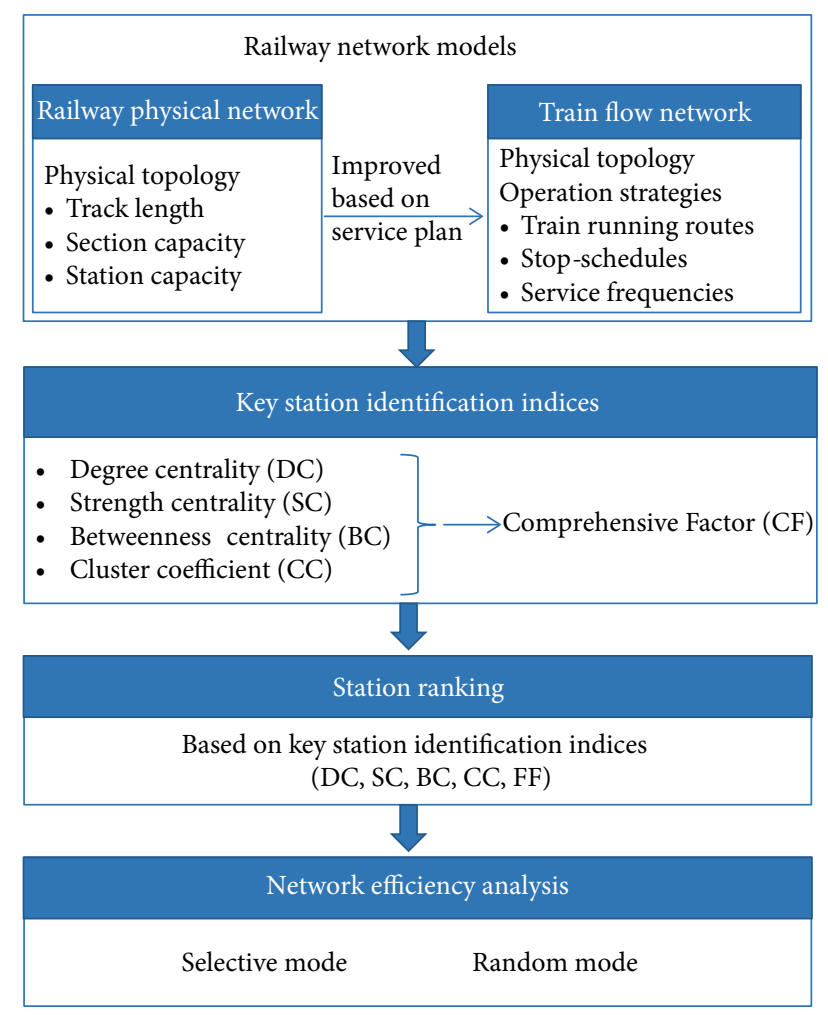

FIGURE 1: Railway network efficiency analysis process.

Key station identification indices can be obtained by evaluating the importance of the stations in a TFN. Stations in the RPN can be ranked based on the evaluated importance of each station. Once key stations are identified, the key station identification indices are then used to analyse network efficiency by the choice of failure modes of stations or the random selection of failure modes of stations in the railway network.

2.1. Railway Physical Network (RPN). The stations can be considered as nodes, and the connection between any two stations can be expressed as an edge in the RPN $[7,16,22,24]$. For example, Figure 2(a) shows a simple RPN in which 8 stations are connected by two rail lines, i.e., A-B-C-D-E (5 stations) and F-G-C-H (4 stations). The station $\mathrm{C}$ is a junction station, and the dark nodes show that these stations are terminal stations that can be as original or destination stations of the trains. The RPN can be expressed as $G_{g}=\left(V_{g}, E_{g}\right)$, where $V_{g}$ is a set of railway stations in the network, and $E_{g}$ is a set of rail tracks. The RPN presents the physical connectivity among the stations in the RPN in which takes the track length, section capacity and station capacity into consideration. The RPN can be used to analyse transportation capacity constraints for train service plan.

2.2. Train Flow Network (TFN). As the stations are represented as nodes in the RPN, if a train has been scheduled to be operated between two stations, this will produce one edge between these two stations as shown in Figure 2(b). The numbers of trains scheduled to stop at any two connected stations determine the weight of edge between the two stations [4]. The total number of edges can be calculated by $C_{n}^{2}=n \times(n-1) / 2$ and $n$ denotes a train that has a total number of stations to stop as

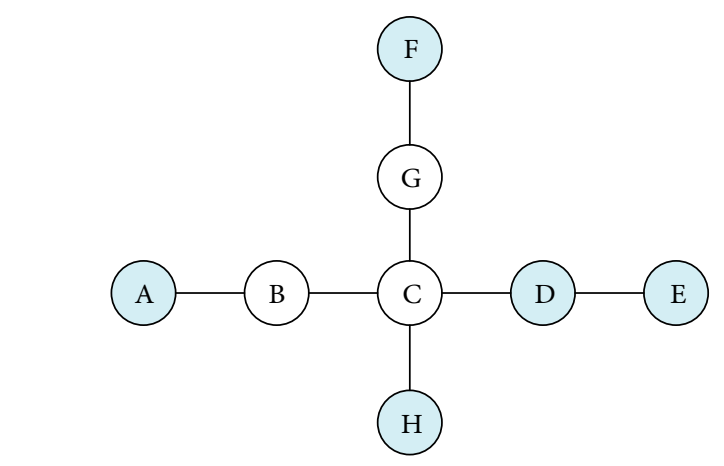

(a)

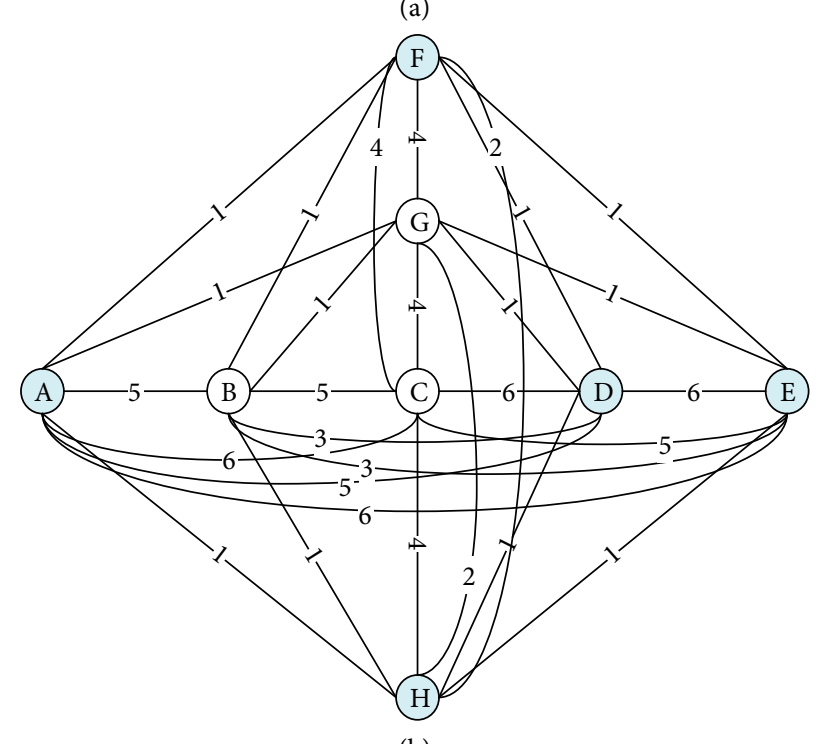

(b)

FIgURE 2: (a) Railway physical network (RPN) and (b) train flow network (TFN).

scheduled. For example, Figure 2(b) shows 8 stations that are connected by 28 edges, i.e., $C_{8}^{2}=(8 \times 7) / 2=28$ between two stations based on the proposed train service plan as shown in Figure 3. The number on each edge presents frequency of trains, for example, number of 5 on edge $A$ to $C$ presents that 5 trains are scheduled running on this edge and stop at station $\mathrm{A}$ and $\mathrm{C}$ as shown in Figure 2(b). The TFN can be expressed as $G_{t}=\left(V_{t}, E_{t}\right)$, where $V_{t}$ is the set of stations that any train can stop at these stations, and $E_{t}$ is a set of edges that creates any two stops at any stations in the RPN. Therefore, the TFN can be developed based on train service plan in which the train stop schedule creates the edges in the RPN, and frequency of trains determines the weight of the edge. Obviously, if the frequency of trains running on an edge is high, the weight of this edge is high.

2.3. RPN and TFN Considerations. As described earlier in this paper, the RPN can be improved by taking the train service plan into consideration to produce the TFN. For example, Figure 3 shows a proposed train service plan which includes 9 stop-schedules, i.e., T1, T2, ..,T9 and frequencies of trains are $2,1,1,3,2,1,1,1$, and 1 , respectively. The nodes as shown in Figure 3 for each stop-schedule means that a train stops at these stations, for example, the stop-schedule of T4(3) 


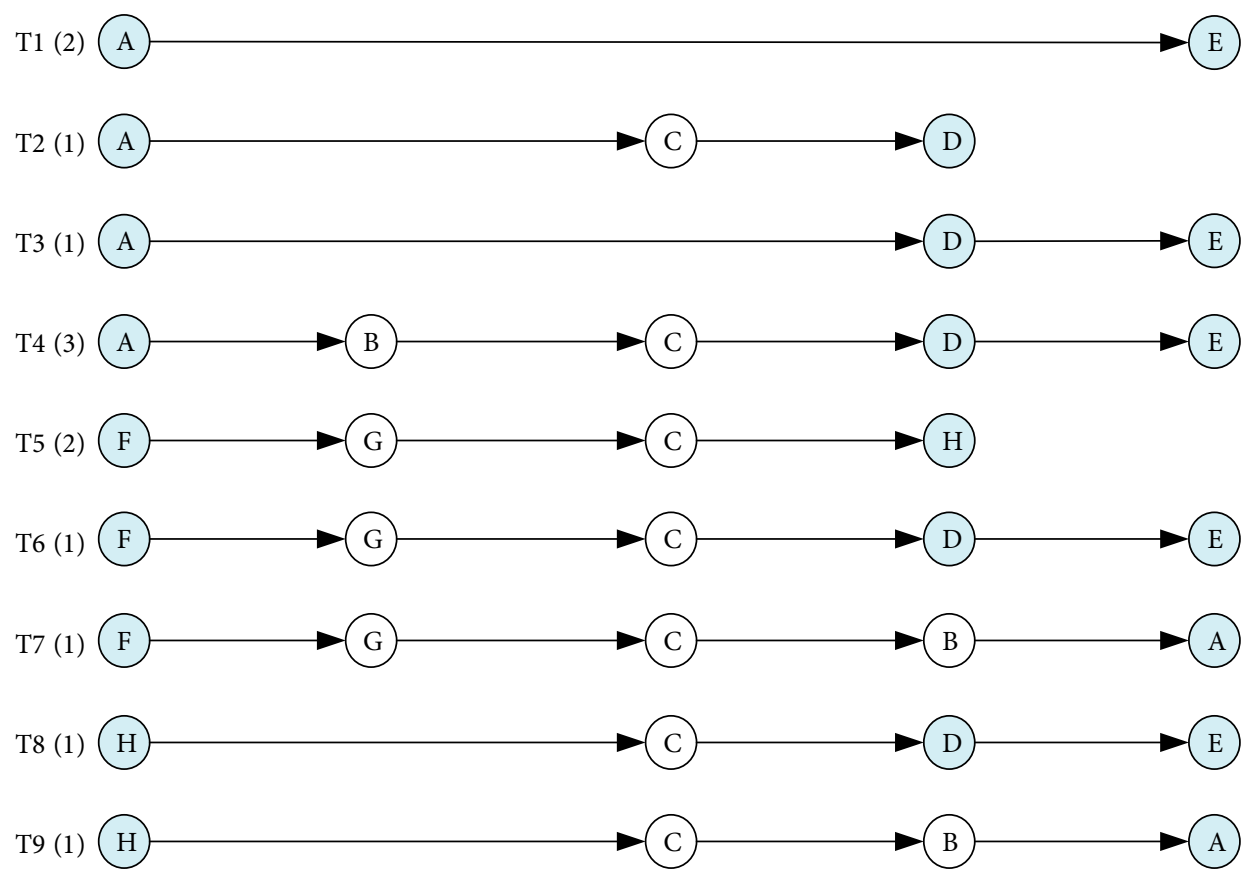

FIGURE 3: Train service plan.

indicates that 3 trains have a same stop-schedule with different departure times, and all of these 3 trains will stop at stations of $\mathrm{A}, \mathrm{B}, \mathrm{C}, \mathrm{D}$, and $\mathrm{E}$. Based on the proposed train service plan, the TFN can be produced as shown in Figure 3 in which train service plan has been taken into consideration.

As can be seen from Figure 2(b), the edge between nodes $\mathrm{A}$ and $\mathrm{B}$ is created by stop-schedules of T4, T7, and T9 as shown in Figure 3, and the weight of edge is the sum of frequencies of these three stop-schedules, i.e., $3+1+1=5$. It should be noted that there is an edge between nodes $\mathrm{F}$ and $\mathrm{C}$ in TFN as shown in Figure 2(b), although there is not a direct connection between F and C in RPN as shown in Figure 2(a). However, the edge between nodes $\mathrm{F}$ and $\mathrm{C}$ in TFN is created by stop-schedules of T5, T6, and T7 as shown in Figure 3, and the edge weight is the sum of frequencies of these three stop-schedules, i.e., $2+1+1=4$ as shown in Figure 2(b). Similarly, other edges based on stop-schedules can be produced, and the weights of edges in TFN can be calculated. In this case, the railway network physical topology in the RPN and the operation strategies such as train running routes, original stations and destination stations, stop-schedules, and service frequencies can be considered together to produce the TFN by taking the relations and weights of edges into account.

\section{Factors Used in Key Station Identification Analysis}

As described in Section 2, the TFN can be developed based on the RPN by taking train service plan into consideration. Key station identification indices can be then calculated based on complex network principles [30], including degree centrality (DC), strength centrality (SC), betweenness centrality (BC), clustering coefficient (CC), and comprehensive factor (CF). These indices are then used to assess the importance of each station in the railway network, which are described as below.

3.1. Degree Centrality (DC). Assume $v_{i}$ denotes the $i$ th node in the TFN, the DC of a node $v_{i}$ is the number of the connections between $v_{i}$ and other nodes in the RPN, which describes the physical connective influence of a node by the number of its neighbour nodes. For example, the DC of Node A is 7 (i.e., $5+1+1=7$ ) as shown in Figure $2(\mathrm{~b})$, which means that 7 Nodes of B, C, D, F, E, G, and H are connected to Node A directly. The DC $k_{i}$ of a node $v_{i}$ in the TFN can be defined as

$$
k_{i}=\sum_{j=1}^{N} n_{i, j},
$$

where $k_{i}$ is the DC of a node, $N$ is the number of the nodes in the RPN, and $n_{i, j}$ is a variable of 0 or 1 , i.e., if there is a connection between nodes $v_{i}$ and $v_{j}$, then $n_{i, j}=1$, otherwise, $n_{i, j}=0$. If a node in the TFN is connected with more edges, it will have a large value of DC $k_{i}$. In other words, the DC of a node describes the reachability of the station.

3.2. Strength Centrality (SC). In the TFN, some of the edges are more important than others that depend on weights of edges. The weight of an edge presents the importance of this edge in the TFN in which it depends on frequencies of train service running on this edge, i.e., the more frequently the edge is used by trains, the more important it is. In this study, the SC is used to describe the weight of each node. For example, the SC of Node $A$ is $25(5+6+5+6+1+1+1=25)$ as shown in Figure 2(b). Assume SC $s_{i}$ of a node $v_{i}$ is the sum of the weights of the edges between $v_{i}$ and other nodes, and it can be defined as

$$
s_{i}=\sum_{j=1}^{N} w_{i, j},
$$


where $s_{i}$ is the SC of a node $v_{i}$, and $w_{i, j}$ is the weight of the edge between node $v_{i}$ and $v_{j}$. The weight $w_{i, j}$ of an edge between nodes $v_{i}$ and $v_{j}$ in the TFN is the number of trains that do stop at the $i$ th and $j$ th stations. The SC of a node also describes the service capability of a specific station, which represents the convenience of the passengers from this station to other stations in the network without any change of the train; in other words, trains can reach more stations from the $i$ th station.

3.3. Betweenness Centrality (BC). The $\mathrm{BC}$ as defined in the complex network principles [5] describes the influence of a node in the network; in this case, it is a station in the RPN. In this study, the $\mathrm{BC}$ relates to the shortest paths from one node to the other one, i.e., from one station to other station. For every pair of nodes, i.e., between two stations in a network, at least there is one shortest path either it has the minimum number of the edges or it has the minimum value of the weights of the edges. A path is defined as from a node $v_{j}$ (i.e., Station $j$ ) to a node $v_{k}$ (i.e., Station $k$ ), which indicates a path passing between two stations in the network based on train service plan $[4,9]$. For example, there are 15 shortest paths between any two nodes in the network as shown in Figure 4(a). Among all the 15 shortest paths, 9 paths pass through node S2 including S1S2, S1-S2-S4, S1-S2-S4-S3, S1-S2-S4-S5, S1-S2-S4-S6, S2-S4, S2-S4-S3, S2-S4-S5, and S2-S4-S6. But 6 paths that are S3-S4, S3-S4-S5, S3-S4-S6, S5-S4, S6-S4, and S5-S4-S6 do not include the node $\mathrm{S} 2$. Therefore, the $\mathrm{BC}$ of the node $\mathrm{S} 2$ is $9 / 15=0.6$.

Assume $\mathrm{BC} b_{i}$ of a node $v_{i}$ in the network without the weight of the edge, which can be calculated by:

$$
b_{i}=\frac{\sum_{j \neq k} g_{j, k}(i)}{\sum_{j \neq k} g_{j, k}},
$$

where $g_{j, k}$ is the number of shortest paths with the minimum number of the edges from a node $v_{j}$ to a node $v_{k}$, and $g_{j, k}(i)$ is the number of shortest paths with the minimum number of the edges, which pass through the node $v_{i}$ from a node $v_{j}$ to a node $v_{k}$.

Similarly, the $\mathrm{BC} b_{i}^{w}$ of a node $v_{i}$ with the weight of the edge is defined as capacity BC, which can be calculated by

$$
b_{i}^{w}=\frac{\sum_{j \neq k} g_{j, k}^{w}(i)}{\sum_{j \neq k} g_{j, k}^{w}}
$$

where $g_{j, k}^{w}$ is the number of shortest paths with the minimum sum value of the weights of the edges from a node $v_{j}$ to a node $v_{k}$, and $g_{j, k}^{w}(i)$ is the number of shortest paths with the minimum sum value of the weights of the edges, in which trains pass through the node $v_{i}$ from a node $v_{j}$ to a node $v_{k}$. The BC reflects the influence of the nodes throughout the network. A node that has a high impact on network efficiency is called as an influential node. For example, the node S2 in Figure 4(a) is such a node because 9 out of 15 short paths go through this node. Therefore, on the basis of the BC analysis, influential nodes can be obtained in different perspectives of connectivity and transportation capacity.

3.4. Clustering Coefficient (CC). The CC represents a node that links with a certain node, and whether those nodes have also

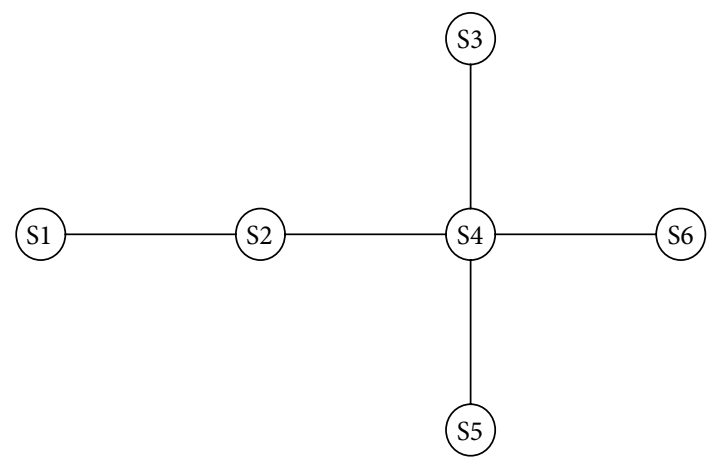

(a)

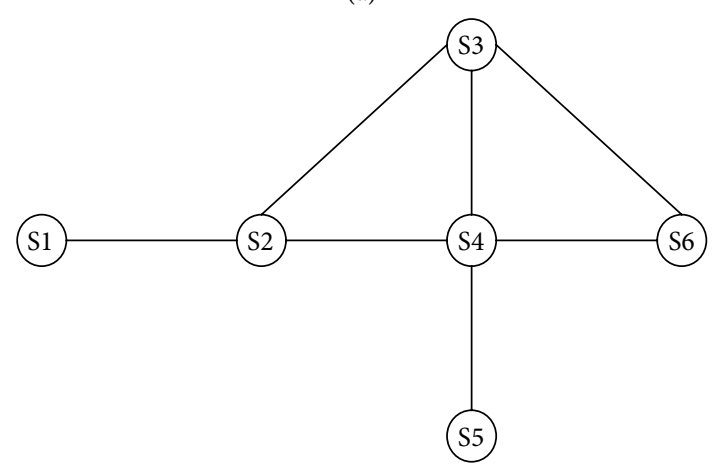

(b)

Figure 4: (a) BC and (b) CC calculation.

connected each other or not. The higher the value of the CC of a node is, the more densely connected nodes will be. The $\mathrm{CC} c_{i}$ of a node $v_{i}$ is defined as

$$
c_{i}=\frac{2 m_{i}}{k_{i}\left(k_{i}+1\right)},
$$

where $c_{i}$ is CC, $k_{i}$ is the DC of the $i$ th node that has a maximum number of edges that equals to $k_{i} \times\left(k_{i}+1\right) / 2$, and $m_{i}$ is the number of the edges that actually exist based on train service plan of the $i$ th node. In other words, the CC represents the influence of the stations in the network. For example, as shown in Figure 4(b), node S4 has actual connections with nodes S2, S3, S5, and S6. In this case, $k_{i}=4$ which consists of 6 edges. i.e., S4-S2, S4-S3, S4-S5, S4-S6, S2-S3, and S3-S6. The maximum number of edges of node S4 is $4 \times(4+1) / 2=10$. However, only 6 connections actually exist in the network and other 4 edges are not actually existing. In other words, these 4 edges indicate that the connections can be created by using other actual connections, for example, node $S 4$ has not an actual link with node S1 but can use the path S2-S1 to create an edge such as S4-S2-S1. Therefore, by using Equation (5), the CC of S4 is $c_{6}=2 \times 6 / 4(4+1)=0.6$. The CC represents the influence of the stations in the network, but it has some problems by using the CC in the ranking importance of stations; for example, in some cases, if some of the nodes have same connections in the network, these nodes will have a same value of the CC. This will be demonstrated in the section of case study.

3.5. Comprehensive Factor (CF). In this study, an important factor of $\mathrm{CF}$ is introduced in order to integrate degree centrality 
(DC), strength centrality (SC), betweenness centrality (BC $b_{i}$ and $b_{i}^{w}$ ) and Clustering Coefficient (CC) in a unique manner for network identification analysis in the TFN. DC, SC, BC $\left(b_{i}\right.$ and $b_{i}^{w}$ ) and CC can be normalized by

$$
\bar{Z}_{i}^{\alpha}=\frac{Z_{i}^{\alpha}-Z_{\alpha}^{\min }}{Z_{\alpha}^{\max }-Z_{\alpha}^{\min }}, \alpha=1,2,3,4,5 .
$$

Then, the CF $C_{i}$ of a node $v_{i}$ can be calculated by

$$
C_{i}=\sum \lambda_{\alpha} \bar{Z}_{i}^{\alpha}, \alpha=1,2,3,4,5,
$$

where $\alpha$ is the number $1,2,3,4$, and 5 , i.e., 1 denotes DC, 2 is $\mathrm{SC}, 3$ is $\mathrm{BC} b_{i}, 4$ is $\mathrm{BC} b_{i}^{w}$, and 5 is CC, $z_{i}^{\alpha}$ represents the values of DC, SC, BC $\left(b_{i}\right.$ and $\left.b_{i}^{w}\right)$, and CC of a node $v_{i}, z_{\alpha}^{\min }$ is the minimum value of $\mathrm{DC}, \mathrm{SC}, \mathrm{BC}\left(b_{i}\right.$ and $\left.b_{i}^{w}\right)$, and $\mathrm{CC}$ of the nodes in the TFN, $z_{\alpha}^{\max }$ is the maximum value of DC, SC, BC $\left(b_{i}\right.$ and $b_{i}^{w}$ ) and CC of the nodes in TFN, $\bar{z}_{i}^{\alpha}$ is normalized value of the node $v_{i}, \lambda_{\alpha}$ is the weight of the $\alpha$, which shows the impact of different $\alpha$ in the CF. The selection of $\lambda_{\alpha}$ depends on the evaluation purposes such as the connectivity and transportation capacity. For example, if DC, SC, BC $\left(b_{i}\right.$ and $\left.b_{i}^{w}\right)$, and CC of the nodes are taken as equally important, then $\lambda$ can be chosen as $1 / 5$. If transportation capacity in the TFN is more important than connectivity in the RFN, then $\lambda$ can be $2 / 5$. Expert judgement and engineering judgement such as the Delphi method [30] can be used in the selection of $\lambda_{\alpha}$.

\section{A New Methodology for Network Efficiency Analysis}

Network reliability can be obtained by the analysis of the characteristics of the network under selective and random station failure modes in the railway network [29]. Selective failure mode will enable network analysts to select stations in the railway network based on the current status of the network and their experience to analyse the network reliability, while random failure mode will enable network analysts to assess network efficiency by selecting stations randomly in the railway network. The network efficiency $E$ and relative network efficiency $R$ are given below to evaluate the reliability of the TFN, which are derived by

$$
\begin{gathered}
E=\frac{2}{N(N-1)} \sum_{i \geq j}^{N} \frac{1}{d_{i, j}}, \\
R=\frac{E}{E_{0}},
\end{gathered}
$$

where $N$ is the total number of nodes in the network after a number of station failures, and $d_{i, j}$ denotes the number of edges in the shortest path between nodes $v_{i}$ and $v_{j}$, which represents the distance between nodes $v_{i}$ and $v_{j}$ (if $v_{i}$ is not connected with $v_{j}$, then $d_{i, j}=+\infty$, and $\left.E=0\right)$, and $E$ is the network efficiency after the failures of selected stations, and $E_{0}$ is the initial network efficiency, $i$ and $j$ denote two different nodes.

\section{Case Study}

5.1. Background. This section presents a case study on network efficiency analysis for a high-speed rail network to demonstrate the application of the proposed methodology for railway network efficiency analysis. The data and information have been collected from the railway industry for one-day train operation, which indicates that in a total of 2487 trains were operated in such a high-speed rail network. Figure 5 shows the established RPN with 485 nodes (i.e., stations in the network). Based on train service plan on the day, the TFN has also been established with the same number of nodes as the RPN has, i.e., 485 nodes, and the number of edges is analysed as described in Section 2.2; in this case, there are 68198 edges which make up the TFN is more complex than the RPN. Since a large amount of data and information in the network has to be analysed, the new methodology described above has been converted into computer code.

5.2. Degree Centrality (DC) Calculation. As stated in Section 3.1, the distribution of DC can be calculated by Equation (1), for convenience, it has been converted into exponential distribution by

$$
P(>s)=1.09 e^{-0.02} \text {. }
$$

Figure 6(a) shows the results of distribution of DC, and the results of exponential distribution of DC are shown in Figure 6(b). As can be seen that only 11 stations that it is about $2.26 \%$ of a total number of stations in the network have a value of DC more than 150. But most of those stations are identified as the hub stations such as Nos. 8, 10, 38, and 48 in Figure 5.

5.3. Strength Centrality (SC) Calculation. The distribution of SC is shown in Figure 7(a), which can be calculated by Equation (2). The results show that only 12 stations (about $4.53 \%$ ) of total number of stations in the network have a value more than 1000 of SC, and 377 stations (about $77.73 \%$ ) of total number of stations in the network have a value less than 400 of SC, which indicates that the distribution of SC of the stations in the TFN is extremely deviated, which represents that only a few stations having high service capacity in the network. In other words, it would be more convenient for the passengers to travel from these high service capacity stations than others. Comparing distributions of DC with SC are shown in Figure 7(b), which has been converted by using power law

$$
s \propto k^{1.242},
$$

where $s$ can be obtained by Equation (1), and $k$ can be calculated by Equation (2). Equation (11) demonstrates if the connectivity of a station in the current transportation operation strategy is $k$, the ability to serve the passengers is $k^{1.242}$. As can be seen in Figure 7(b), the SC is increased faster than the DC, in other words, the transportation capacity of a station is growing faster than the growth of connectivity.

5.4. Betweenness Centrality $(B C)$ Calculation. The distributions of BC $b_{i}$ and $b_{i}^{w}$ (i.e., with/without the weight of an edge) in the 


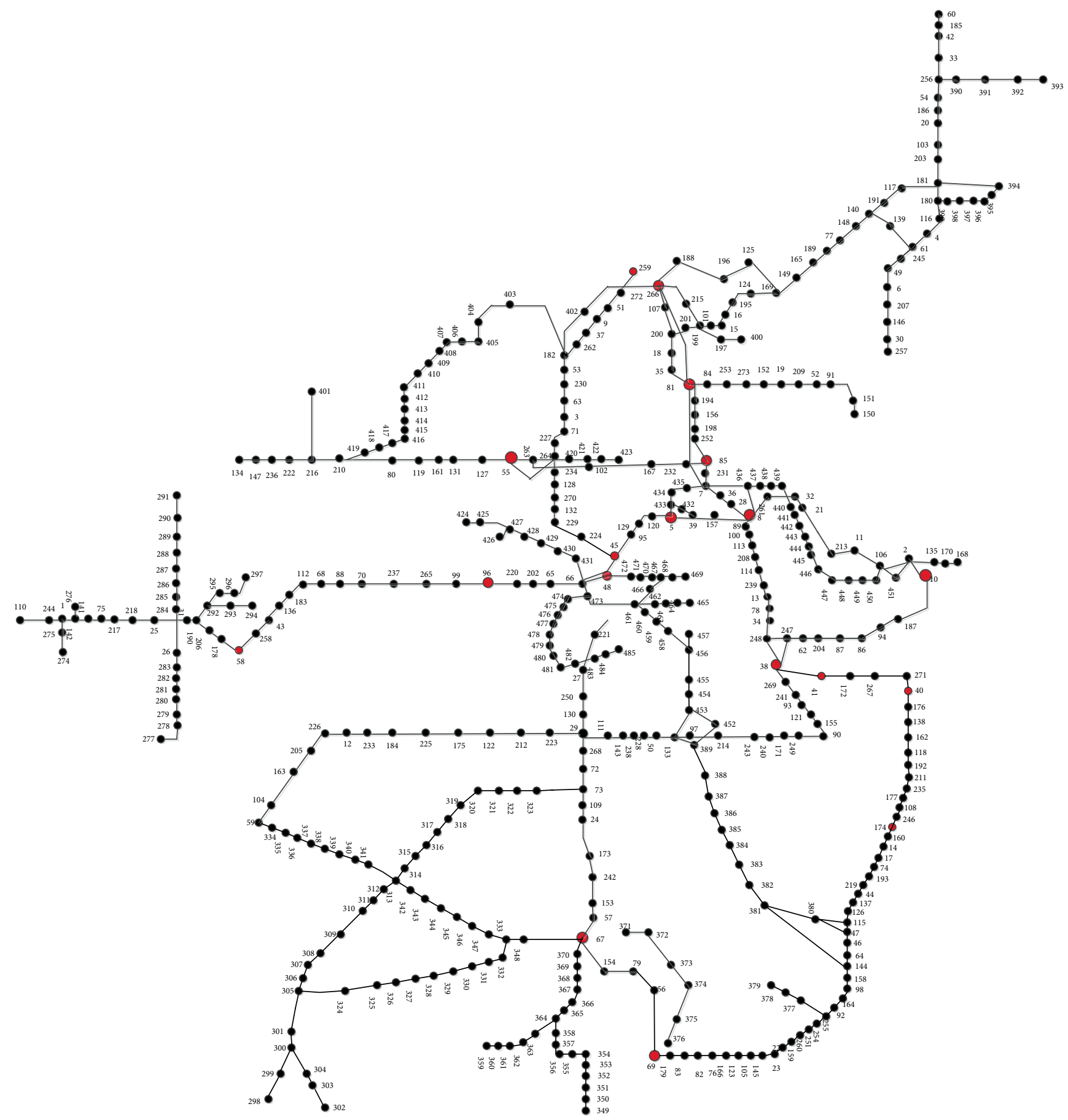

FIgURE 5: High-speed railway physical network in China.

TFN are given in Table 1 and shown in Figure 8, which can be calculated by Equations (3) and (4). Most of the stations have a small value of probability of $b_{i}$ and $b_{i}^{w}$. Value ranges of $\mathrm{BC} b_{i}$ and $\mathrm{BC} b_{i}^{w}$ in Table 1 are presented as percentage. Only 5 stations have a large value of $b$, which shows that $1.8 \%$ of total stations in the railway network with a value between 0.05730 and 0.06548 . In other words, these 5 stations are important and contribute significantly to the efficiency of the TFN.
5.5. Clustering Coefficient Calculation. The CC can be calculated by using Equation (5). Figure 9(a) shows the distribution of the CC in the cast study. The average of CC in the TFN is 0.697 , which demonstrates high aggregation characteristics of the TFN, i.e., stations within the railway network are closely connected. The relationship between the CC and DC of each node is shown in Figure 9(b). As can be seen from Figure 9(b) that, obviously, a node with high CC has a low value of DC. In other words, the 


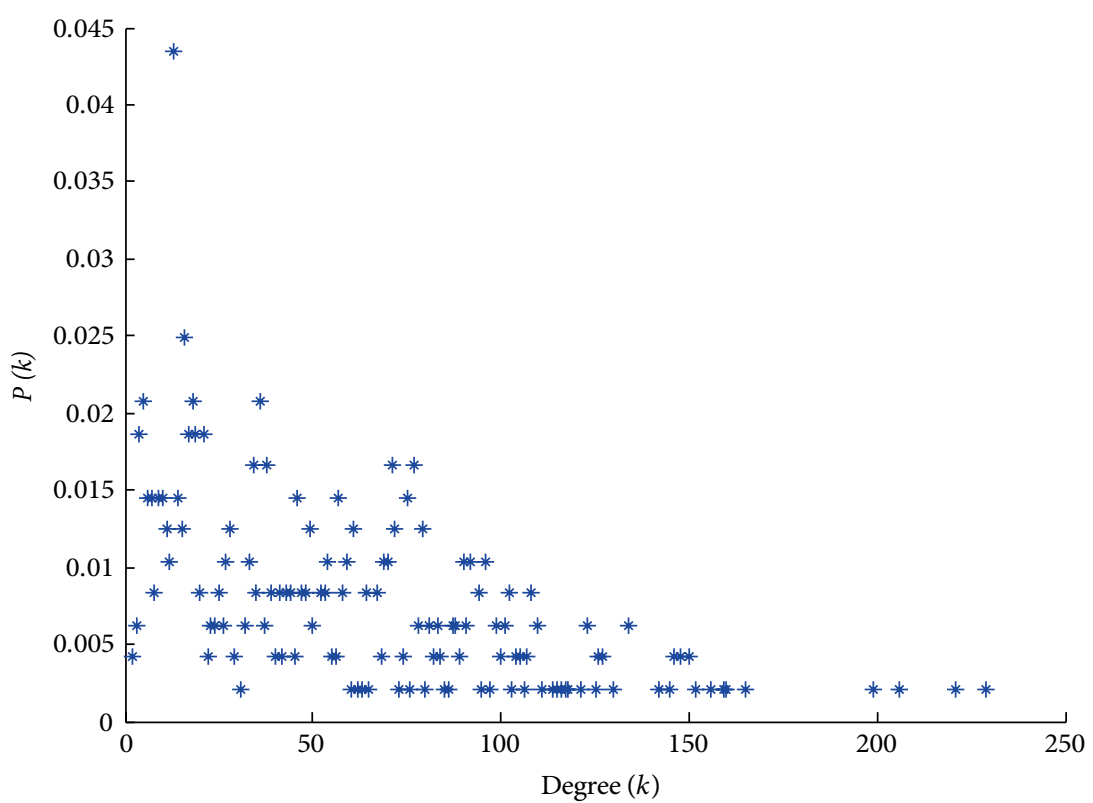

(a)

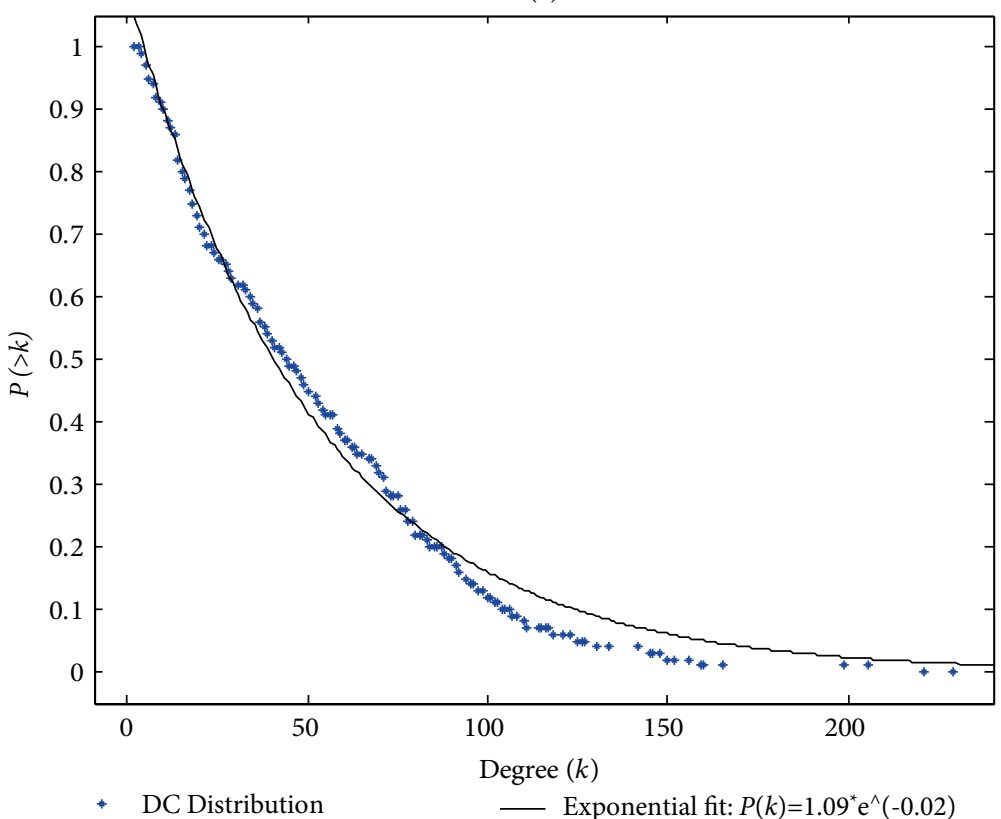

(b)

Figure 6: (a) Distribution and (b) exponential distribution of DC.

lower value of the DC the station has, the greater value of the CC that the station has.

5.6. Comprehensive Factor. The CF can be calculated by Equations (6) and (7). Table 2 shows the results and station important rankings of top 20 stations based on their values of the CF, and these stations are also presented in Figure 5 denoted by red spots. Table 2 also shows rankings based on values of DC, SC, TBC, CBC, and CC, respectively. For example, in the column of station (DC), the rankings are based on the values of the DC of the stations, which station identity numbers are given that can be found in Figure 5 and the values of the DC of the stations. In comparison of the rankings based on values of the $\mathrm{CF}$ of the stations with the rankings based on values of the $\mathrm{DC}, \mathrm{SC}, \mathrm{BC}\left(b_{i}, \mathrm{BC} b_{i}^{w}\right), \mathrm{CBC}$ and $\mathrm{CC}$ of stations, as can be seen that rankings of stations are different. The reason is that the evaluations of DC, SC, $\mathrm{BC}\left(b_{i}\right.$ and $\left.b_{i}^{w}\right)$ and CC have different focuses as discussed in Sections 3.1-3.5. However, the $\mathrm{CF}$ addresses all of issues that focuses on DC, SC, BC $b_{i}, \mathrm{BC} b_{i}^{w}$ and CC have; in other words, $\mathrm{CF}$ not only takes the railway network physical topology, but also the operation strategies into consideration such as train running routes, original stations and destination stations, stop-schedules, and service frequencies. Therefore, by using 


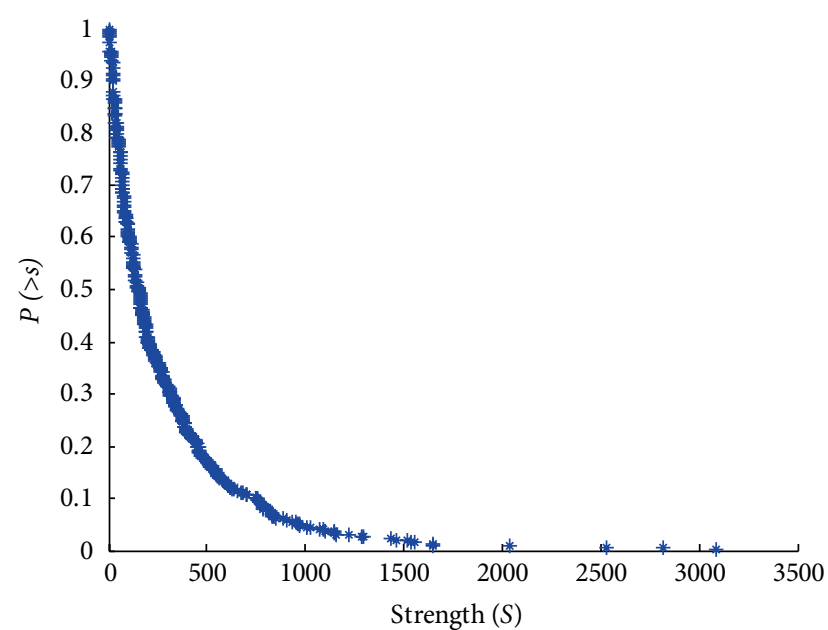

(a)

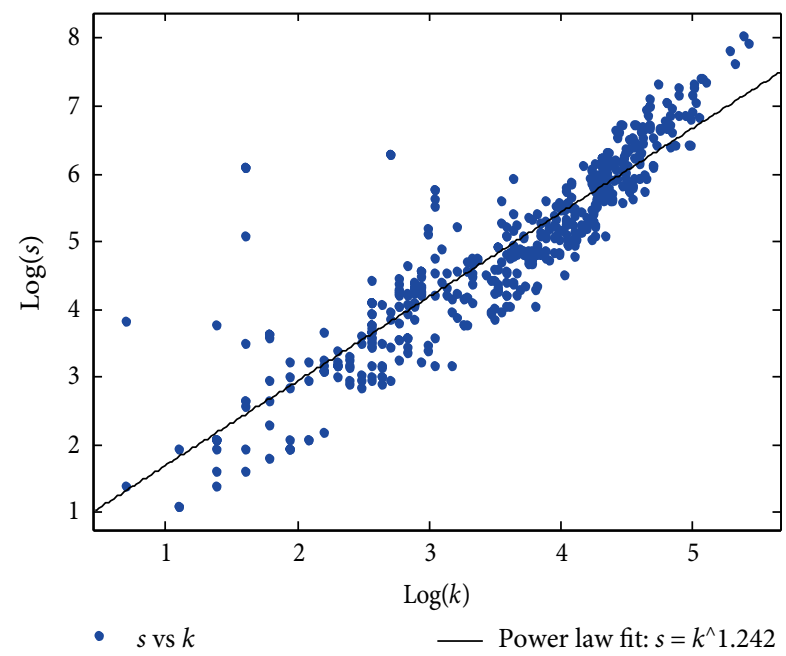

(b)

FIgure 7: (a) Distribution of SC and (b) DC versus SC.

TABLE 1: Distributions of BC $b_{i}$ and $b_{i}^{w}$.

\begin{tabular}{lcc}
\hline $\begin{array}{l}\text { Value range of BC } b_{i} \\
\text { and BC } b_{i}^{w}\end{array}$ & Probability of BC $b_{i}$ & Probability of BC $b_{i}^{w}$ \\
\hline $0.00000-0.00005$ & 0.168498 & 0.161172 \\
$0.00005-0.00030$ & 0.131868 & 0.146520 \\
$0.00030-0.00100$ & 0.194139 & 0.201465 \\
$0.00100-0.00307$ & 0.197802 & 0.201465 \\
$0.00307-0.00501$ & 0.124542 & 0.10989 \\
$0.00501-0.00603$ & 0.036630 & 0.032967 \\
$0.00603-0.01037$ & 0.058608 & 0.058608 \\
$0.01037-0.02206$ & 0.040293 & 0.040293 \\
$0.02206-0.05730$ & 0.029304 & 0.029304 \\
$0.05730-0.06548$ & 0.018315 & 0.018315 \\
\hline
\end{tabular}

$\mathrm{CF}$ in the assessment railway network efficiency, more reliable results can be obtained. This can be demonstrated that, for example, in an emergency, a train may be delayed or cancelled; in this case, the RPN is not changed, but the weights and

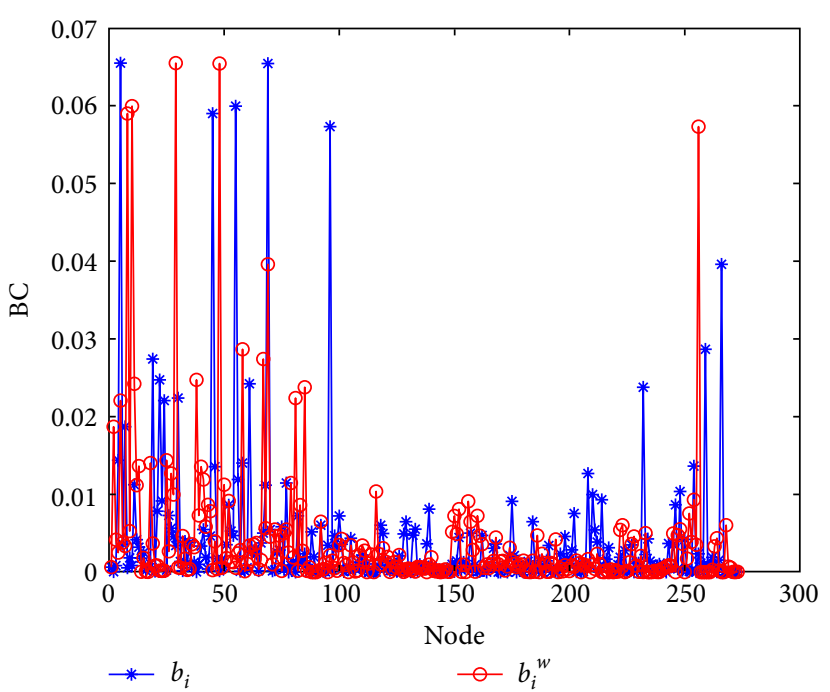

FIGURE 8: Distributions of $\mathrm{BC} b_{i}$ and $\mathrm{BC} b_{i}^{w}$.

edges of the TFN are changed, and application of a single $\mathrm{DC}$ or SC or $\mathrm{BC} b_{i}$, or $\mathrm{BC} b_{i}^{w}$ or CC cannot provide reliable results; $\mathrm{CF}$ takes all the factors that $\mathrm{DC}, \mathrm{SC}, \mathrm{BC} b_{i}, \mathrm{BC} b_{i}^{w}$ , and CC used in the consideration; therefore, by using $\mathrm{CF}$, more reliable results can be obtained. The results of this case study have been further confirmed by the industry from their observation in the railway network [5].

Additionally, based on the results produced by the evaluation using CF, as can be seen that most of nodes (i.e., stations) with the high values of CF are in the central and eastern regions in the railway network as shown in Figure 5. This is particularly true because this is also confirmed that these areas have a high economic development with high populations and high level of demand of transportation. However, it should be noted that it is not all of these top 20 stations are in the areas with high economic development and high populations. For example, although station Nos. 11, 40, 41, 58, 96, and 174 have higher values of $\mathrm{CF}$, these stations only have one rail line pass through as shown in Figure 5. In order words, these stations (i.e., nodes) have lower physical connectivity in the RPN but have a higher transportation capacity because the frequency of trains using these stations is high. In other words, more trains are scheduled to use these stations. Another interesting finding is that the stations Nos. 259 and 266 in the capital city, are ranked in the places of $17^{\text {th }}$ and $14^{\text {th }}$, and this is because there are four stations in the capital city to decentralize transport pressure. Other issues should be noted as stated in Section 3.4, in some cases, the application of the CC in the ranking importance of stations is not effective. In this case, as can be seen from Table 2, the top 20 stations have a same value of the CC because these stations have a same number of connections with other stations in the network.

5.7. Network Efficiency Analysis. In this case, the average network distance of RPN and TFN can be obtained as 30.84 and 2.89 by analysing the network. In other words, the average network distance 30.84 in the RPN represents that a passenger travels from an original station to a destination 


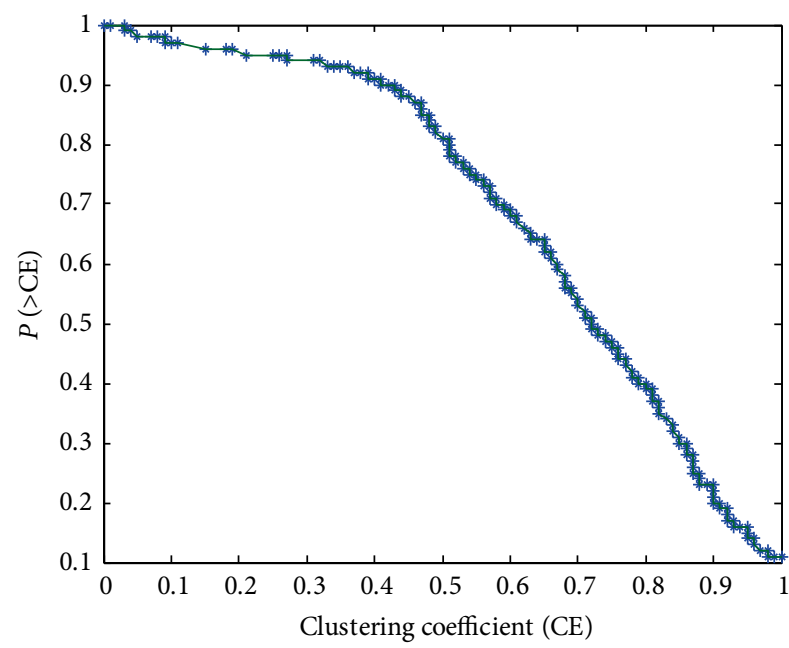

(a)

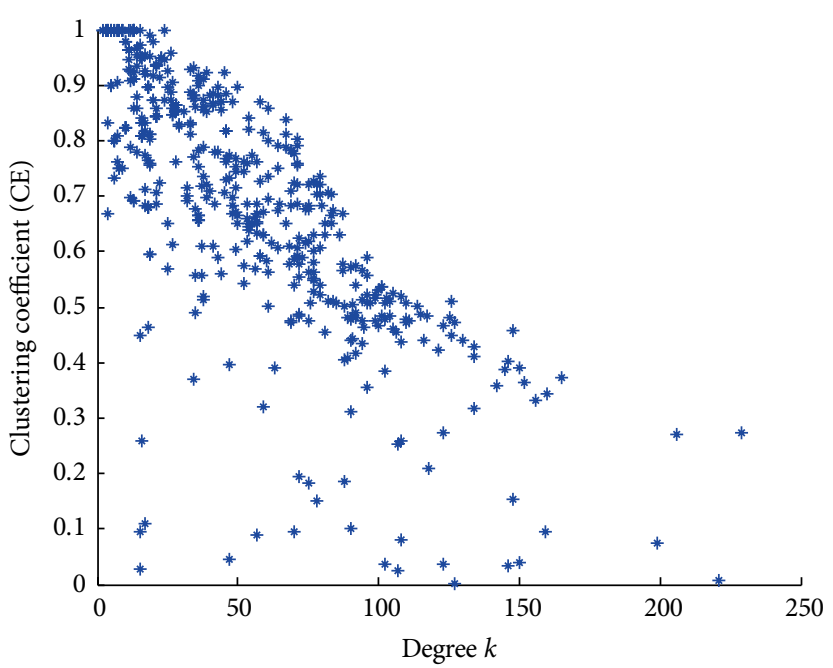

(b)

Figure 9: (a) Distributions of CC and (b) CC versus DC.

TABLE 2: Ranking of top 20 stations.

\begin{tabular}{|c|c|c|c|c|c|c|}
\hline Rank & Station (DC) & Station (SC) & Station $\left(\mathrm{BC} b_{i}\right)$ & Station $\left(\mathrm{BC} b_{i}^{w}\right)$ & Station $(\mathrm{CF})$ & Station $(\mathrm{CC})$ \\
\hline 1 & $8(229.0)$ & $8(3088.0)$ & $5(0.065478)$ & $29(0.065478)$ & $8(2.909347)$ & \multirow{20}{*}{$\begin{array}{c}88,89,90,197,209,215, \\
217,219,234,235,246,250,257 \\
258,259,260,261,266,267,272 \\
\text { have a same value of the CC }\end{array}$} \\
\hline 2 & $10(221.0)$ & $10(2813.0)$ & $69(0.065424)$ & $48(0.065423)$ & $10(2.856245)$ & \\
\hline 3 & $38(206.0)$ & $38(2528.0)$ & $55(0.059942)$ & $10(0.059942)$ & $5(2.594738)$ & \\
\hline 4 & $48(199.0)$ & $29(2037.0)$ & $45(0.058993)$ & $8(0.058993)$ & $69(2.511870)$ & \\
\hline 5 & $85(165.0)$ & $40(1652.0)$ & $96(0.057304)$ & $256(0.057304)$ & $48(2.282593)$ & \\
\hline 6 & $5(160.0)$ & $81(1648.0)$ & $266(0.039598)$ & $69(0.039598)$ & $29(2.281926)$ & \\
\hline 7 & $29(159.0)$ & 85 (1556.0) & $259(0.028668)$ & $58(0.028668)$ & $38(2.179361)$ & \\
\hline 8 & $40(156.0)$ & $48(1537.0)$ & $19(0.027414)$ & $67(0.027414)$ & 45 (1.962085) & \\
\hline 9 & $11(152.0)$ & $174(1516.0)$ & $22(0.024731)$ & $38(0.024731)$ & 55 (1.914219) & \\
\hline 10 & $41(150.0)$ & $25(1462.0)$ & $61(0.024227)$ & $11(0.024227)$ & $96(1.842426)$ & \\
\hline 11 & $81(150.0)$ & $5(1430.0)$ & $232(0.023781)$ & $85(0.023781)$ & $85(1.718422)$ & \\
\hline 12 & $2(148.0)$ & $11(1297.0)$ & $30(0.022393)$ & $81(0.022393)$ & $11(1.692670)$ & \\
\hline 13 & $43(148.0)$ & $12(1287.0)$ & $24(0.022068)$ & $5(0.022068)$ & $40(1.651933)$ & \\
\hline 14 & $13(146.0)$ & $248(1219.0)$ & $7(0.018700)$ & $2(0.018700)$ & $266(1.626548)$ & \\
\hline 15 & $12(146.0)$ & $67(1152.0)$ & $4(0.014389)$ & $25(0.014389)$ & $58(1.615441)$ & \\
\hline 16 & $39(145.0)$ & $2(1149.0)$ & $58(0.014031)$ & $18(0.014031)$ & $81(1.601190)$ & \\
\hline 17 & $83(142.0)$ & $13(1147.0)$ & $254(0.013638)$ & $13(0.013638)$ & $259(1.473424)$ & \\
\hline 18 & $67(134.0)$ & $41(1098.0)$ & $46(0.013559)$ & $40(0.013559)$ & $67(1.467032)$ & \\
\hline 19 & $160(134.0)$ & $126(1089.0)$ & $208(0.012686)$ & $27(0.012686)$ & 41 (1.460257) & \\
\hline 20 & $69(134.0)$ & $44(1068.0)$ & $56(0.011917)$ & $41(0.011917)$ & $174(1.423473)$ & \\
\hline
\end{tabular}

station that at an average the passenger needs to pass through about $31 \quad(30.84 \approx 31)$ stations, and the average network distance 2.89 in the TFN represents the average number of trains that a passenger changes trains during the travel in the railway network. In this case, the passenger needs to change $2(2.89-1=1.89 \approx 2)$ trains at an average during the travel from an original station to a destination station in the railway network. The network efficiency can be calculated by Equation (8) as described in Section 4, which shows that the network efficiency of the TFN is 2.10 , and the RPN is 0.06. This demonstrates that the network efficiency of the
TFN is higher than the RPN, in other words, this represents physical connectivity of the railway network in this case is not very dense, but it has a high service capacity and convenient transportation services. The relative network efficiency $R$ can be calculated by Equation (9). Figure 10 shows distributions of $R$ under different station failure modes based on selective and random failure modes. Selective mode 5 shows the percentage of failures of top 20 stations, which presents that relative network efficiency is declined sharply until $10 \%$ of top 20 station failure. When $40 \%$ of top 20 station failures, efficiency $R$ of the railway network is near the zero. Random 


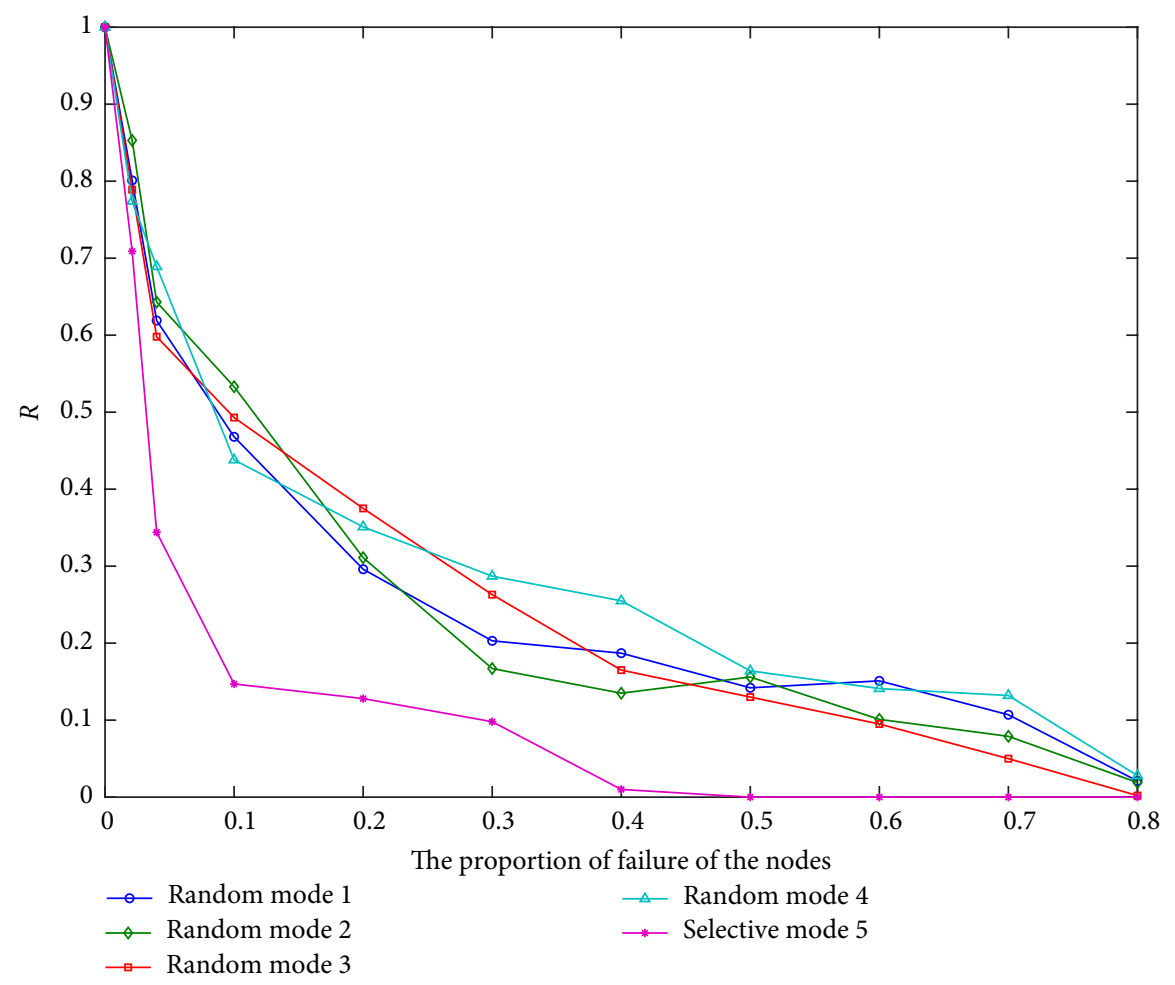

FIgURE 10: Distribution of $R$ under station failure modes.

models 1, 2, 3, and 4 show efficiency $R$ under randomly selected percentage of the station failures in the network. The following assumptions are made to implement the selection of random modes $[4,9]$ :

(i) All stations in the network have an equal opportunity to be selected.

(ii) Each station may be/may not be selected at every time or not.

(iii) Selections of stations start from $10 \%$ of station failures to $80 \%$ of station failures in every random mode.

As can be seen from Figure 10, when $80 \%$ of stations in the network fail to provide service in random modes, the network efficiency $R$ is near the zero. Therefore, in comparison results of selective mode with random modes, the identified top 20 stations as key stations in the network have high impacts on the network efficiency, which require more attention in operation and further development in the railway network. Based on the results produced by the railway network efficiency analysis, recommendations for improvement and optimisation of a railway network can be made, which should address two aspects in the planning and operation.

5.7.1. Railway Network Infrastructure Planning. The network efficiency should be considered in the future railway infrastructure planning by taking the economic and demographic factors into consideration. In this case, some of stations with a high CF in the network only have one railway line passing through, and these stations should consider a new rail line to be constructed, which will not only balance the distribution of the key stations in the network and relieves the transportation pressure but also help to improve the network efficiency.

The combination of RPN and TFN indicates that some stations are located in the same city, for instance, 4 stations in the capital city. Some hub links between these stations should be allocated in the future, which can not only to improve the physical connectivity of the network and transportation services but also improve the efficiency of the whole network in different failure modes.

5.7.2. Railway Transportation Operation. According to the efficiency analysis of the railway network, once the key stations are failed or lost service capacity, the connectivity and efficiency of the overall network would drop down rapidly. To ensure the railway network to provide service, it is recommended to establish operation and maintenance strategies to protect these key stations in the railway network in case of emergency, for example, accidents and incidents, and extreme weather. Furthermore, service capacity can be improved by optimising operation scheme with the constraints of the existing RPN. As described in Section 3.1, the higher $k$ is in the network, the higher service capacity of the network can provide. Therefore, a better operation scheme can also increase efficiency of the railway network.

\section{Conclusions}

The paper presented a new method to analyse the efficiency of the railway network by identifying the key stations based 
on the two network models of RPN and TFN. Both physical network topology and dynamic operation strategies are considered in this method. Considering the key stations, railway network efficiency can be analysed under selective and random failure modes. A real case study on a high-speed railway network is presented to demonstrate the application of the proposed method. In this case, the key stations can be identified based on the CF in which connectivity, transportation capacity and local influence are taken into consideration. The results show that the identified key stations in the railway network play an important role in improving the overall railway network efficiency, which provide useful information to railway designers, engineers, operators and maintainers to operate and maintain railway network effectively and efficiently.

As stated above, the proposed new method considers physical network topology and dynamic operation strategies in the railway network efficiency analysis process. As suggestions, the following aspects in future work may need to be considered to obtain information on availability and stability of the railway network. Research should address (1) application of the proposed method by taking railway transportation organization strategy into physical network consideration to establish a vehicle flow network for railway transportation organization availability analysis, (2) methods to maintain key stations within a railway network as normal service in an emergency because of an accident or incident occurred, and (3) methods to assess the existing networks by increasing number of new stations while improving efficiency of the railway network.

\section{Data Availability}

The readers can find the data used to support the findings of this study are included within the article.

\section{Conflicts of Interest}

The authors declare that they have no conflicts of interest.

\section{Acknowledgments}

This work describes herein is part of research projects funded by National Key Research and Development Programme of China on "Safety and Security Technology of High-speed Railway System (Grant No. 2016YFB1200401), National Natural Science Foundation of China on "Research on Theory and Methodology of Train Operation Adjustment in Urban Regional Railway Network under Special Conditions (Grant No. 71701010), and Highways England on "New Methodology for Maintenance Decision Making of Structures (Grant No. 546037-PMRB13). Their support is gratefully acknowledged.

\section{References}

[1] M. An, Y. Qin, L. M. Jia, and Y. Chen, "Aggregation of group fuzzy risk information in the railway risk decision making process," Safety Sciences, vol. 82, pp. 18-28, 2016.
[2] M. An, W. Lin, and A. Stirling, "Fuzzy-reasoning-based approach to qualitative railway Risk assessment," Proceedings of the Institution of Mechanical Engineers, Part F: Journal of Rail and Rapid Transit, vol. 220, no. 2, pp. 153-167, 2006.

[3] M. An, S. Huang, and C. J. Baker, "Railway risk assessment - the FRA and FAHP approaches: a case study of shunting at Waterloo depot," Proceedings of the Institution of Mechanical Engineers, Part F: Journal of Rail and Rapid Transit, vol. 221, pp. 1-19, 2007.

[4] X. Meng, W. Xiang, and L. Wang, "Controllability of train service network," Mathematical Problems in Engineering, vol. 2015, Article ID 631492, 2015.

[5] M. Ouyang, L. Zhao, L. Hong, and Z. Pan, "Comparisons of complex network based models and real train flow model to analyse Chinese railway vulnerability," Reliability Engineering and System Safety, vol. 123, pp. 38-46, 2014.

[6] A. Nagurney and Q. Qiang, "A network efficiency measure with application to critical infrastructure networks," Global Optimization, vol. 2008, no. 40, pp. 261-275, 2008.

[7] L. Wang, M. An, Y. Zhang, and K. Rana, "Railway network reliability analysis based on key station identification using complex network theory: a real-world case study of high-speed rail network," in International Research Conference 2017 (IRC 2017): Shaping Tomorrow's Built Environment, 11-12 October 2017, University of Salford, UK, pp. 395-408, 2017.

[8] L. Li, L. Jia, Y. Wang, and J. Li, "Reliability evaluation for complex system based on connectivity reliability of network model," in Proceedings of 2015 International Conference on Logistics, Informatics and Service Sciences (LISS), IEEE, Barcelona, Spain, pp. 1-5, 27-29 July, 2015.

[9] M. Saleh, Y. Esa, and A. Mohamed, "Applications of complex network analysis in electric power systems," Energies, vol. 11, no. 6, pp. 1-16, 2018.

[10] H. Zenil, N. A. Kiani, and J. Tegnér, "Methods of information theory and algorithmic complexity for network biology," 2014, http://arxiv.org/abs/1401.3604.

[11] L. C. Yin, H. Kretschmer, R. A. Hanneman, and Z. Y. Liu, "Connection and stratification in research collaboration: an analysis of the COLLNET network," Information Processing \& Management, vol. 42, no. 6, pp. 1599-1613, 2006.

[12] M. A. Koseoglu, "Mapping the institutional collaboration network of strategic management research: 1980-2014," Scientometrics, vol. 109, no. 1, pp. 203-226, 2016.

[13] D. P. Chassin and C. Posse, "Evaluating North American electric grid reliability using the Barabási-Albert network model," Physica A: Statistical Mechanics and its Applications, vol. 355, no. 2-4, pp. 667-677, 2005.

[14] X. L. An, L. Zhang, Y. Z. Li, and J. G. Zhang, "Synchronization analysis of complex networks with multi-weights and its application in public traffic network," Physica A: Statistical Mechanics and its Applications, vol. 412, pp. 149-156, 2014.

[15] A. Vázquez, R. Pastor-Satorras, and A. Vespignani, "Large-scale topological and dynamical properties of the internet," Physical Review E: Statistical, Nonlinear, and Soft Matter Physics, vol. 65, no. 6, Article ID 066130, 2002.

[16] P. Dey, R. Mehra, F. Kazi, S. Wagh, and N. M. Singh, "Impact of topology on the propagation of cascading failure in power grid," IEEE Transactions on Smart Grid, vol. 7, no. 4, pp. 1970-1978, 2016.

[17] E. Zio and G. Sansavini, "Modeling interdependent network systems for identifying cascade-safe operating margins," IEEE Transactions on Reliability, vol. 60, no. 1, pp. 94-101, 2011. 
[18] S. V. Buldyrev, R. Parshani, G. Paul, H. E. Stanley, and S. Havlin, "Catastrophic cascade of failures in interdependent networks," Nature, vol. 464, no. 7291, pp. 1025-1028, 2010.

[19] H. P. Ren, J. Song, R. Yang, M. S. Baptista, and C. Grebogi, "Cascade failure analysis of power grid using new load distribution law and node removal rule," Physica A: Statistical Mechanics and its Applications, vol. 442, pp. 239-251, 2016.

[20] J. Yan, H. He, and Y. Sun, "Integrated security analysis on cascading failure in complex networks," IEEE Transactions on Information Forensics and Security, vol. 9, no. 3, pp. 451-463, 2014.

[21] S. Dunn and S. Wilkinson, "Hazard tolerance of spatially distributed complex networks," Reliability Engineering and System Safety, vol. 157, pp. 1-12, 2017.

[22] X. Xu, J. Hu, F. Liu, and L. Liu, "Scaling and correlations in three bus-transport networks of China," Physica A: Statistical Mechanics and its Applications, vol. 374, no. 1, pp. 441-448, 2007.

[23] S. Porta, P. Crucitti, and V. Latora, "The network analysis of urban streets: a dual approach," Physica A: Statistical Mechanics and its Applications, vol. 369, no. 2, pp. 853-866, 2006.

[24] G. Bagler, "Analysis of the airport network of India as a complex weighted network," Physica A: Statistical Mechanics and its Applications, vol. 387, no. 12, pp. 2972-2980, 2008.

[25] L. Dall'Asta, A. Barrat, M. Barthélemy, and A. Vespignani, "Vulnerability of weighted networks," Journal of Statistical Mechanics: Theory and Experiment, vol. 2006, no. 4, 2006.

[26] R. Guidotti, P. Gardoni, and Y. Chen, "Network reliability analysis with link and nodal weights and auxiliary nodes," Structural Safety, vol. 65, pp. 12-26, 2017.

[27] Y. Qian, B. Wang, Y. Xue, J. Zeng, and N. Wang, "A simulation of the cascading failure of a complex network model by considering the characteristics of road traffic conditions," Nonlinear Dynamics, vol. 80, no. 1-2, pp. 413-420, 2015.

[28] S. Chen, X. Zou, Q. Xu, and H. Lv, "Invulnerability optimization of Chinese railway traffic network for suppressing cascading failure," Journal of Information and Computational Science, vol. 11, no. 5, pp. 1501-1509, 2014.

[29] S. Lin, L. Jia, Y. Wang, Y. Qin, and M. Li, "Reliability study of bogie system of high-speed train based on complex networks theory," in Proceedings of the 2015 International Conference on Electrical and Information Technologies for Rail Transportation, Springer Berlin Heidelberg, pp. 117-124, 2016.

[30] A. L. Barabási and R. Albert, "Emergence of scaling in random networks," Sciences, vol. 286, no. 5439, pp. 509-512, 1999. 


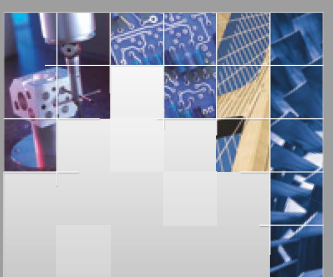

\section{Enfincering}
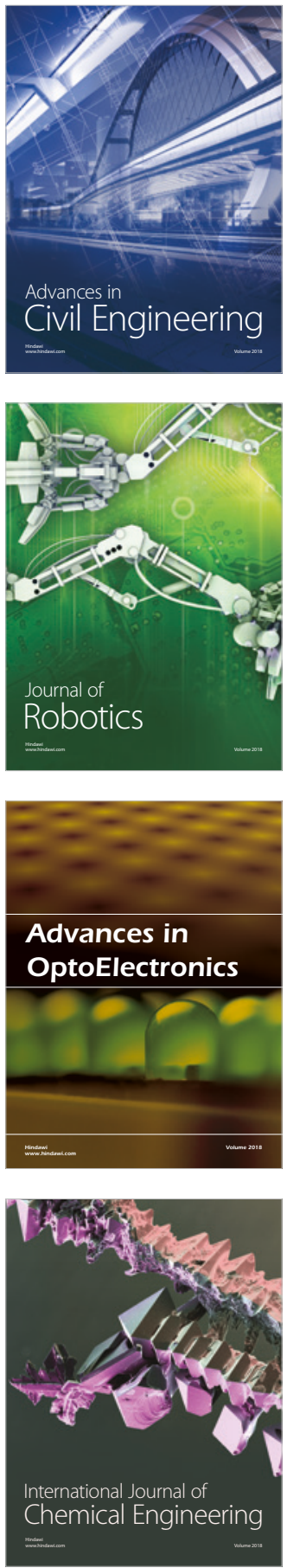

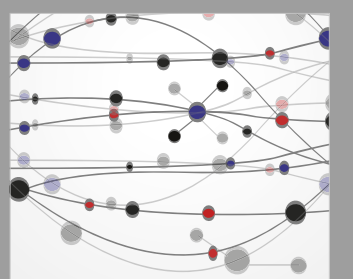

\section{Rotating \\ Machinery}

The Scientific World Journal

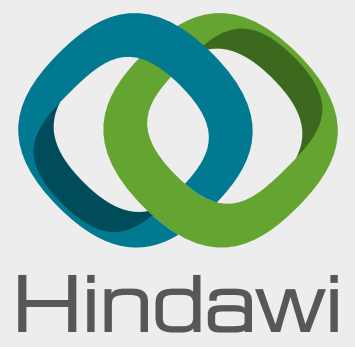

Submit your manuscripts at

www.hindawi.com
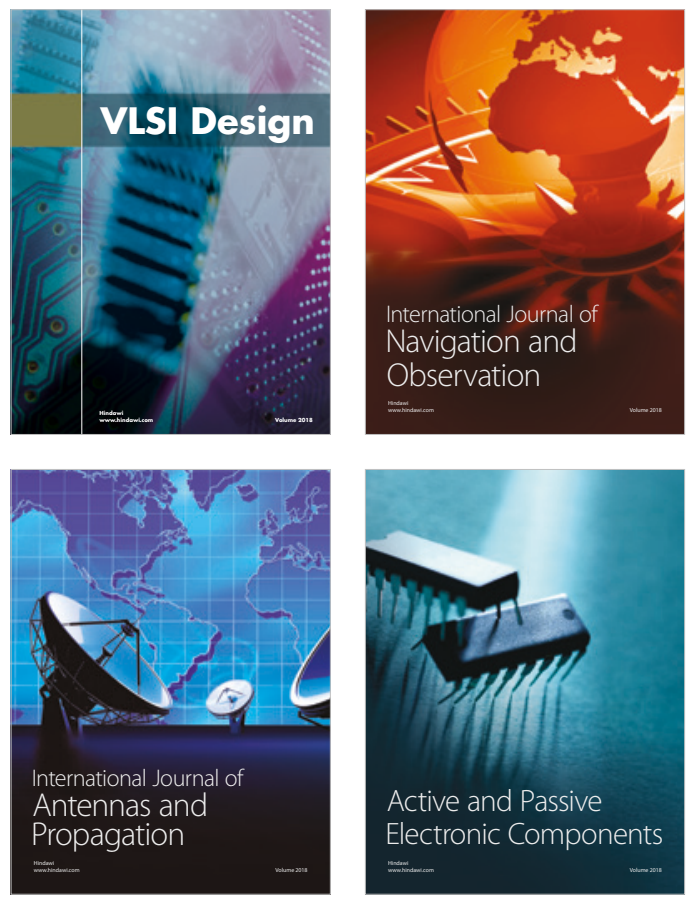
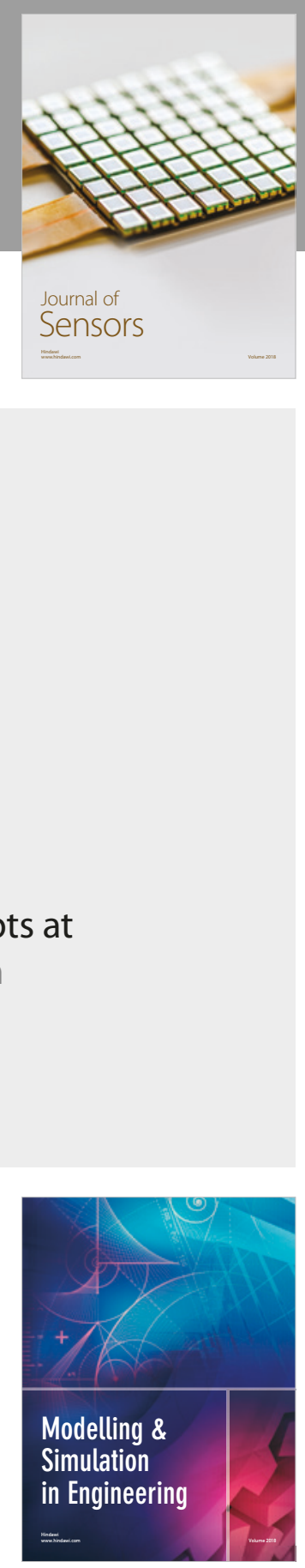

\section{Advances \\ Multimedia}
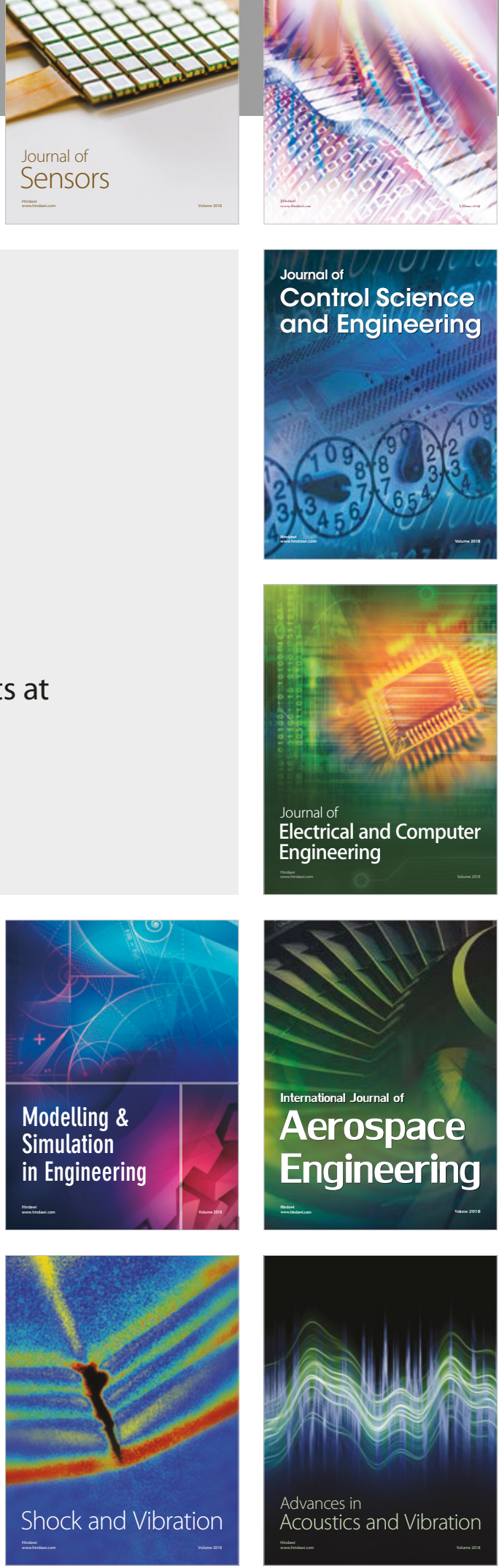